\title{
The (signless Laplacian) spectral radii of connected graphs with prescribed degree sequences
}

\author{
Muhuo Liu* \\ ${ }^{1}$ Department of Mathematics \\ South China Agricultural University \\ Guangzhou, China \\ ${ }^{2}$ School of Mathematical Science \\ Nanjing Normal University \\ Nanjing, China \\ liumuhuo@163.com
}

Submitted: Jun 28, 2012; Accepted: Nov 21, 2012; Published: Dec 6, 2012

Mathematics Subject Classifications: 05C35, 05C75, 05C05

\begin{abstract}
In this paper, some new properties are presented to the extremal graphs with largest (signless Laplacian) spectral radii in the set of all the connected graphs with prescribed degree sequences, via which we determine all the extremal tricyclic graphs in the class of connected tricyclic graphs with prescribed degree sequences, and we also prove some majorization theorems of tricyclic graphs with special restrictions.
\end{abstract}

Keywords: Spectral radius, signless Laplacian spectral radius, degree sequence, majorization

\section{Introduction}

Throughout the paper, $G$ denotes a connected undirected simple graph with $n$ vertices and $m$ edges, unless specified otherwise. If $m=n+c-1$, then $G$ is called a $c$-cyclic graph. In particular, when $c=0,1,2$ or 3 , then $G$ is called a tree, unicyclic graph, bicyclic graph or tricyclic graph, respectively. As usual, denote $N_{G}(v)$ the neighbor set of vertex $v$ in

\footnotetext{
${ }^{*}$ Supported by National Natural Science Foundation of China (Nos. 11071088, 11201156), and the Foundation for Distinguished Young Talents in Higher Education of Guangdong, China (LYM10039), and Project of Graduate Education Innovation of Jiangsu Province (No. CXZZ12-0378).
} 
$G$, and let $d_{G}(v)$ be the degree of $v$. When there is no confusion, we simplify $N_{G}(v)$ and $d_{G}(v)$ as $N(v)$ and $d(v)$, respectively. If $d(v)=1, v$ is called a pendant vertex.

Let $A(G)$ be the adjacency matrix of $G$, and let $D(G)$ be the diagonal matrix whose $(i, i)$-entry is $d\left(v_{i}\right)$. The signless Laplacian matrix of $G$ is $Q(G)=D(G)+A(G)$. We use the notations $\rho(G)$ and $\mu(G)$ to denote the spectral radius and signless Laplacian spectral radius of $G$, respectively, namely, $\rho(G)$ and $\mu(G)$ are, respectively, equal to the largest eigenvalues of $A(G)$ and $Q(G)$.

When $G$ is connected, by the Perron-Frobenius Theorem of non-negative matrices (see e. g. [4]), $\rho(G)$ and $\mu(G)$ have multiplicity one and there exists a unique positive unit eigenvector corresponding to $\rho(G)$, and there also exists a unique positive unit eigenvector corresponding to $\mu(G)$. In this paper, we use $f=\left(f\left(v_{1}\right), \ldots, f\left(v_{n}\right)\right)^{T}$ to indicate the unique positive unit eigenvector corresponding to $\rho(G)$ or $\mu(G)$, and call $f$ the Perron vector of $G$.

If $d_{i}=d\left(v_{i}\right)$ for $i=1,2, \ldots, n$, then we call the sequence $\pi=\left(d_{1}, d_{2}, \ldots, d_{n}\right)$ the degree sequence of $G$. Throughout this paper, we enumerate the degrees in non-increasing order, i.e., $d_{1} \geqslant d_{2} \geqslant \ldots \geqslant d_{n}$. Let $\Gamma(\pi)$ define the class of connected graphs with a prescribed degree sequence $\pi$, and let $\mathbf{S}(\pi)$ be the class of connected tricyclic graphs with a prescribed tricyclic degree sequence $\pi$. In the coming discussion, we call $G$ an extremal graph if $G$ has largest spectral radius or signless Laplacian spectral radius of $\Gamma(\pi)$.

Suppose $\pi=\left(d_{1}, d_{2}, \ldots, d_{n}\right)$ and $\pi^{\prime}=\left(d_{1}^{\prime}, d_{2}^{\prime}, \ldots, d_{n}^{\prime}\right)$ are two non-increasing integer sequences, we write $\pi \triangleleft \pi^{\prime}$ if and only if $\pi \neq \pi^{\prime}, \sum_{i=1}^{n} d_{i}=\sum_{i=1}^{n} d_{i}^{\prime}$, and $\sum_{i=1}^{j} d_{i} \leqslant \sum_{i=1}^{j} d_{i}^{\prime}$ for all $j=1,2, \ldots, n$. Such an ordering is sometimes called majorization. Suppose that $G$ and $G^{\prime}$ are the extremal graphs of $\Gamma(\pi)$ and $\Gamma\left(\pi^{\prime}\right)$, respectively. We say that the spectral radii (respectively, signless Laplacian spectral radii) of $G$ and $G^{\prime}$ satisfy the majorization theorem if $\pi \triangleleft \pi^{\prime}$ implies that $\rho(G)<\rho\left(G^{\prime}\right)$ (respectively, $\mu(G)<\mu\left(G^{\prime}\right)$ ).

The work on determining the graph which has largest spectral radius among some class of graphs, can be traced back to 1985 when Brualdi and Hoffman investigated the maximum spectral radius of the adjacency matrix of a (not necessarily connected) graph in the set of all graphs with a given number of vertices and edges. Their work was followed by other people, in the connected graph case as well as in the general case.

In this line, the unique extremal graph of $\Gamma(\pi)$ was characterized when $\Gamma(\pi)$ are restricted on trees, unicyclic graphs and/or bicyclic graphs, respectively $[1,2,5,11,16,17]$, and the (signless Laplacian) spectral radii of extremal graphs were proved to satisfy the majorization theorem when $\Gamma(\pi)$ are restricted on trees, unicyclic graphs and/or bicyclic graphs, respectively $[2,5,6,8,16,17]$. Furthermore, Liu et al. [9] found that the majorization theorem is a good tool to deal with Cvetković's problem, asked how to classify and order graphs according to their spectral radii [3]. Unfortunately, this method (namely, the tool of majorization theorem) cannot be applied to deal with Cvetković's problem for the spectral radii of tricyclic graphs, since a counterexample to the majorization theorem of tricyclic graphs was discovered by Liu et al. [10].

In this paper, some new properties are presented to the extremal graphs of $\Gamma(\pi)$, and all the extremal tricyclic graphs of $\mathbf{S}(\pi)$ will be determined. Furthermore, we also verify some majorization theorems of tricyclic graphs with special restrictions. 
The rest of this paper is organized as follows: We first give some new properties to the extremal graphs of $\Gamma(\pi)$ in Section 2, via which we characterize all the extremal tricyclic graphs of $\mathbf{S}(\pi)$ in Sections 3. Finally, some majorization theorems of tricyclic graphs with special restrictions are given in Section 4.

\section{Extremal graphs of $\Gamma(\pi)$}

Let $G-u v$ denote the graph obtained from $G$ by deleting the edge $u v \in E(G)$. Similarly, denote by $G+u v$ the graph obtained from $G$ by adding an edge $u v \notin E(G)$.

Lemma 1. $[15,16]$ Let $u, v$ be two vertices of the connected graph $G$, and $w_{1}, w_{2}, \ldots, w_{k}$ $(1 \leqslant k \leqslant d(v))$ be some vertices of $N(v) \backslash(N(u) \cup\{u\})$. Let $G^{\prime}=G+w_{1} u+\cdots+w_{k} u-$ $w_{1} v-\cdots-w_{k} v$. Suppose $f$ is the Perron vector of $G$. If $f(u) \geqslant f(v)$, then $\rho\left(G^{\prime}\right)>\rho(G)$ and $\mu\left(G^{\prime}\right)>\mu(G)$.

Corollary 2. Suppose $G$ is an extremal graph of $\Gamma(\pi)$ and $f$ is the Perron vector of $G$. If $d(v)>d(u)$, then $f(v)>f(u)$. Moreover, if $f(v)=f(u)$, then $d(v)=d(u)$.

Proof. Suppose that there exist vertices $v$ and $u$ such that $d(v)>d(u)$, but $f(v) \leqslant f(u)$. Since $G$ is connected, we may suppose that $P_{u v}$ is a shortest path from $u$ to $v$. Note that $d(v)-d(u)=k>0$. Then, there exist vertices $\left\{w_{1}, w_{2}, \ldots, w_{k}\right\} \subseteq N(v) \backslash(N(u) \cup\{u\})$ such that $w_{1}, w_{2}, \ldots, w_{k} \notin V\left(P_{u v}\right)$. Let $G^{\prime}=G+u w_{1}+\cdots+u w_{k}-v w_{1}-\cdots-v w_{k}$. Then, $G^{\prime} \in \Gamma(\pi)$. Since $f(v) \leqslant f(u), \rho\left(G^{\prime}\right)>\rho(G)$ and $\mu\left(G^{\prime}\right)>\mu(G)$ by Lemma 1 , contradicting the choice of $G$.

Lemma 3. ([4], P. 492-493) Suppose $M=M_{n \times n}$ is a symmetric, nonnegative matrix, $y$ is an $n$-tuple positive vector, $\alpha$ and $\beta$ are two nonnegative real numbers. If $\alpha y \leqslant M y \leqslant \beta y$, then $\alpha \leqslant \lambda \leqslant \beta$, where $\lambda$ is the largest eigenvalue of $M$. Furthermore, $\alpha y<M y$ implies that $\alpha<\lambda$, and $M y<\beta y$ implies that $\lambda<\beta$.

Proposition 4. Let $G=(V, E)$ be a connected graph such that $u x \in E, v y \in E, u v \notin E$, $x y \notin E$, and let $f$ be the Perron vector of $G$. Let $G^{\prime}=G+u v+x y-u x-v y$ (not necessary simple). Suppose $G^{\prime}$ is not connected and $G^{*}$ is a connected component of $G^{\prime}$ so that $u v \in E\left(G^{*}\right)$ and $x y \notin E\left(G^{*}\right)$. If $f(u) \geqslant f(y)$ and $f(v) \geqslant f(x)$, then $\rho\left(G^{*}\right) \geqslant \rho(G)$ and $\mu\left(G^{*}\right) \geqslant \mu(G)$. Moreover, $\rho\left(G^{*}\right)=\rho(G)$ (respectively, $\mu\left(G^{*}\right)=\mu(G)$ ) if and only if $f(u)=f(y)$ and $f(v)=f(x)$.

Proof. Let $f_{1}$ be a vector which is the restriction of $f$ on $V\left(G^{*}\right)$. Since $u v \in E\left(G^{*}\right)$ and $x y \notin E\left(G^{*}\right), \rho(G) f_{1} \leqslant A\left(G^{*}\right) f_{1}$ and $\mu(G) f_{1} \leqslant Q\left(G^{*}\right) f_{1}$. By Lemma 3 we can conclude that $\rho\left(G^{*}\right) \geqslant \rho(G)$ and $\mu\left(G^{*}\right) \geqslant \mu(G)$, where both equalities hold if and only if $f(u)=f(y)$ and $f(v)=f(x)$.

By Lemma 3, we can restate Lemma 3 of [2] and Lemma 3.3 of [16] as follows. 
Lemma 5. $[2,16]$ Let $G=(V, E)$ be a connected graph such that $u x \in E$, vy $\in E$, $u v \notin E, x y \notin E$. Let $G^{\prime}=G+u v+x y-u x-v y$. Suppose $f$ is the Perron vector of $G$. If $f(u) \geqslant f(y)$ and $f(v) \geqslant f(x)$, then $\rho\left(G^{\prime}\right) \geqslant \rho(G)$ and $\mu\left(G^{\prime}\right) \geqslant \mu(G)$. Moreover, $\rho\left(G^{\prime}\right)=\rho(G)$ (respectively, $\mu\left(G^{\prime}\right)=\mu(G)$ ) if and only if $f(u)=f(y)$ and $f(v)=f(x)$.

Corollary 6. Let $G$ be an extremal graph of $\Gamma(\pi)$ and let $f$ be the Perron vector of $G$. Suppose $u x \in E, v y \in E, u v \notin E, x y \notin E$. Let $G^{\prime}=G+u v+x y-u x-v y$. If $G^{\prime} \in \Gamma(\pi)$, then

(1) $f(u)>f(y)$ if and only if $f(v)<f(x)$;

(2) $f(u)=f(y)$ if and only if $f(v)=f(x)$, and $f(u)=f(y)$ (respectively, $f(v)=f(x)$ ) if and only if $G^{\prime}$ is also an extremal graph of $\Gamma(\pi)$.

Definition 7. Let $G$ be a connected graph and $f$ be the Perron vector of $G$. A wellordering $v_{1} \prec v_{2} \prec \cdots \prec v_{n}$ of $V(G)$ is called a $B F S$-ordering if the following hold for all vertices $u, v \in V(G)$ :

(i) $d\left(v_{1}\right) \geqslant d\left(v_{2}\right) \geqslant \cdots \geqslant d\left(v_{n}\right), f\left(v_{1}\right) \geqslant f\left(v_{2}\right) \geqslant \cdots \geqslant f\left(v_{n}\right)$ and $h\left(v_{1}\right) \leqslant h\left(v_{2}\right) \leqslant$ $\cdots \leqslant h\left(v_{n}\right)$, where $h\left(v_{i}\right)$ is the distance between $v_{i}$ and $v_{1}$.

(ii) If $v \in N(u) \backslash N(x), y \in N(x) \backslash N(u)$ such that $h(u)=h(x)=h(v)-1=h(y)-1$, then $f(u)>f(x)$ if and only if $f(v)>f(y)$, and $f(u)=f(x)$ if and only if $f(v)=f(y)$.

Furthermore, if $V(G)$ has a $B F S$-ordering, then we call $G$ a $B F S$-graph.

Suppose $v_{1} \prec v_{2} \prec \cdots \prec v_{n}$ is a $B F S$-ordering of $V(G)$. Denote by $\operatorname{dist}(u, v)$ the distance between $u$ and $v$ in $G$, and let $h(v)=\operatorname{dist}\left(v_{1}, v\right)$. Set $A_{i}=\left\{v: \operatorname{dist}\left(v_{1}, v\right)=i\right\}$. In some literatures (for instance, $[2,5]$ ), $A_{i}$ is also called the $i$-th layer vertices of $G$. Clearly, $A_{0}=\left\{v_{1}\right\}$ and $A_{1}=N\left(v_{1}\right)$. We write $u \equiv v$ if and only if we can interchange the positions of $u$ and $v$ in $\prec$ to obtain another $B F S$-ordering of $V(G)$.

Lemma 8. $[2,17]$ Suppose $G$ is an extremal graph of $\Gamma(\pi)$, and $f$ is the Perron vector of $G$. Then, $V(G)$ has a well-ordering $v_{1} \prec v_{2} \prec \cdots \prec v_{n}$ such that $d\left(v_{1}\right) \geqslant d\left(v_{2}\right) \geqslant \cdots \geqslant d\left(v_{n}\right)$, $f\left(v_{1}\right) \geqslant f\left(v_{2}\right) \geqslant \cdots \geqslant f\left(v_{n}\right)$ and $h\left(v_{1}\right) \leqslant h\left(v_{2}\right) \leqslant \cdots \leqslant h\left(v_{n}\right)$.

Lemma 9. Suppose $G$ is an extremal graph of $\Gamma(\pi)$, and $f$ is the Perron vector of $G$. Then, $V(G)$ has a BFS-ordering such that

(1) if $h(u)=h(v)$, then $u \equiv v$ if and only if $f(u)=f(v)$.

(2) if $h(u)=h(v)=h(w), u \equiv v$ and $v \equiv w$, then $u \equiv w$.

Proof. We first show that $V(G)$ has a $B F S$-ordering. By Lemma 8, $V(G)$ has a wellordering $v_{1} \prec v_{2} \prec \cdots \prec v_{n}$ so that Definition $7(i)$ holds. Thus, it suffices to deduce that Definition 7 (ii) also holds. Let $G^{\prime}=G+u y+x v-u v-x y$. Then, $G^{\prime} \in \Gamma(\pi)$, and hence Definition 7 (ii) follows from Corollary 6. 
We secondly prove (1). Without loss of generality, suppose that $u \prec v$ in the ordering $\prec$. Clearly, $u \equiv v$ implies that $f(u)=f(v)$, since $f(u) \geqslant f(v)$ by Definition $7(i)$. Thus, it suffices to show that $f(u)=f(v)$ also implies that $u \equiv v$.

Now, we suppose that $f(u)=f(v)$. We interchange the positions of $u$ and $v$ in the ordering $\prec$ to obtain a new ordering $\prec^{\prime}$. Since $f(u)=f(v), d(u)=d(v)$ by Corollary 2 . So, $\prec^{\prime}$ satisfies $(i)$ of Definition 7 because $h(u)=h(v)$. Furthermore, $\prec^{\prime}$ clearly satisfies (ii) of Definition 7, since $\prec$ satisfies (ii). So, (1) holds.

Finally, we turn to prove (2). Since $u \equiv v$ and $v \equiv w, f(u)=f(v)=f(w)$ by (1). Now, (1) implies that $u \equiv w$. Thus, (2) holds.

Theorem 10. Let $G$ be an extremal graph of $\Gamma(\pi)$ and $f$ be the Perron vector of $G$. Then, $V(G)$ has a BFS-ordering $\prec$ such that

(1) if $h(u)=h(v)=h(w), u \prec v \prec w, u w \in E(G)$ and $u v \notin E(G)$, then $x \in N(w)$ holds for any $x \in N(v) \backslash\{w\}$ with $u \prec x$, and there must exist some $y \prec u$ such that $y \in N(v) \backslash N(w)$. Furthermore, if $h\left(v_{2}\right)=h(v)=h(w), v_{2} \prec v \prec w$, then $v_{2} w \in E(G)$ implies that $v_{2} v \in E(G)$.

(2) if $f\left(v_{1}\right)>f\left(v_{2}\right)$, then $h(u)<h(v)$ implies that $f(u)>f(v)$.

Proof. We first prove (1). By Lemma 9, $V(G)$ has a $B F S$-ordering $\prec$. Assume, to the contrary, that the result is not true. Let $u$ and $w$ be the least vertices and $v$ be the last vertex in the ordering $\prec$ of $V(G)$ such that $h(u)=h(v)=h(w), u \prec v \prec w, u w \in E(G)$, $u v \notin E(G)$ and there exists some vertex $x \in N(v) \backslash\{w\}$ with $u \prec x$, but $x \notin N(w)$. We may suppose that $v \not \equiv w$ (Otherwise, we will consider the new $B F S$-ordering $\prec^{\prime}$ of $V(G)$ obtained from $\prec$ by interchanging the positions of $v$ and $w)$. So, $f(v)>f(w)$ by Lemma 9 (1) and Definition $7(i)$. Let $G^{\prime}=G+u v+w x-u w-v x$. By Corollary 6 (1), it follows that $f(u)<f(x)$, contradicting $(i)$ of Definition 7 .

If $y \in N(w)$ holds for every $y \in N(v)$ with $y \prec u$, since $u \in N(w) \backslash N(v)$ and $x \in N(w)$ holds for any $x \in N(v) \backslash\{w\}$ with $u \prec x$, we have $d(v)<d(w)$, contradicting Definition $7(i)$.

Thus, $V(G)$ has a $B F S$-ordering $\prec$ such that (1) holds. Now, we turn to show (2). It suffices to show that $f(u)>f(v)$ whenever $u \in A_{j}$ and $v \in A_{j+1}$ holds for $j \geqslant 0$ by induction. The result clearly follows for $j=0$ by the condition $f\left(v_{1}\right)>f\left(v_{2}\right)$. Now, we assume that the result already holds for $0 \leqslant j \leqslant k-1$, and we will prove that the result also follows for $j=k$.

Suppose that there exist two vertices, say $u$ and $v$, such that $u \in A_{k}, v \in A_{k+1}$ and $f(u) \leqslant f(v)$. Since $h(u)<h(v)$, we have $f(u)=f(v)$ by Definition $7(i)$, and hence $d(u)=d(v)$ by Corollary 2. Let $P_{u v_{1}}$ be a shortest path from $v_{1}$ to $u$, and let $P_{v v_{1}}$ be a shortest path from $v_{1}$ to $v$. Choose $x \in V\left(P_{u v_{1}}\right)$ such that $x \in N(u)$. Then, $x v \notin E(G)$ and $h(x)=h(u)-1$.

Suppose $u \in V\left(P_{v v_{1}}\right)$. Then $d(v)=d(u) \geqslant 2$ and $u v \in E\left(P_{v v_{1}}\right)$. Since $d(v)=d(u) \geqslant 2$ and $x \in N(u) \backslash N(v)$, there exists some $y \in N(v) \backslash\{u\}$ such that $y \notin N(u)$. Since $u \in N(v) \cap V\left(P_{v v_{1}}\right), h(y) \geqslant h(u)=h(x)+1$. If $h(y)>h(u)$, by Definition $7(i)$ we have $f(u) \geqslant f(y)$. By the induction hypothesis and $h(x)=k-1<k=h(u), f(x)>f(u)$. 
Thus, $f(x)>f(y)$. If $h(y)=h(u)$, since $h(x)=k-1<k=h(u)=h(y)$, by the induction hypothesis we have $f(x)>f(y)$. So, we get $f(x)>f(y)$ whenever $h(y)>h(u)$ or $h(y)=h(u)$. Let $G^{\prime}=G+v x+u y-u x-v y$. Since $u x \in E(G)$, uv $\in E(G)$ and vy $\in E(G)$, we can conclude that $G^{\prime} \in \Gamma(\pi)$. Now, Corollary 6 (1) implies that $f(v)<f(u)$, a contradiction.

Suppose $u \notin V\left(P_{v v_{1}}\right)$. Choose $y \in N(v) \cap V\left(P_{v v_{1}}\right)$ such that $h(y)=h(u)$. Thus, $f(x)>f(y)$ by the induction hypothesis and $h(x)=k-1<k=h(u)=h(y)$. If $y u \notin E(G)$, let $G^{\prime}=G+v x+u y-u x-v y$. Then, $f(v)<f(u)$ by Corollary 6 (1), a contradiction. If $y u \in E(G)$, since $x \in N(u) \backslash N(v)$ and $d(u)=d(v)$, there exists some $z \in N(v) \backslash\{u\}$ such that $z \notin N(u)$. Since $h(z) \geqslant h(y)=h(u), f(x)>f(z)$ by the induction hypothesis and Definition $7(i)$. Let $G^{\prime}=G+x v+u z-u x-v z$. Now, Corollary $6(1)$ implies that $f(v)<f(u)$, a contradiction.

In the following, if $G$ is an extremal graph of $\Gamma(\pi)$, we always suppose that $V(G)=$ $\left\{v_{1}, v_{2}, \ldots, v_{n}\right\}$ has a $B F S$-ordering $\prec$ such that $\prec$ satisfies the conclusion of Theorem 10 .

Lemma 11. Let $G$ be an extremal graph of $\Gamma(\pi)$, and uv be an edge on a cycle of $G$. Suppose $P=w_{1} \cdots w_{s+1}$ is a path of $G$, and $f\left(w_{s+1}\right)<\min \{f(u), f(v)\}$, where $f$ is the Perron vector of $G$. If there exists some $j \in\{1,2, \ldots, s\}$ such that $u w_{j} \notin E(G)$, $u w_{t} \notin E(G)$ and $v w_{t} \notin E(G)$ hold for every $t \in\{j+1, \ldots, s+1\}$, then $f(v)>f\left(w_{j}\right)$.

Proof. To the contrary, suppose that $f(v) \leqslant f\left(w_{j}\right)$. Let $G^{\prime}=G+u w_{j}+v w_{j+1}-u v-$ $w_{j} w_{j+1}$. Then, $G^{\prime} \in \Gamma(\pi)$. By Lemma $5, f(u) \leqslant f\left(w_{j+1}\right)$. Let $G^{\prime \prime}=G+u w_{j+2}+v w_{j+1}-$ $u v-w_{j+1} w_{j+2}$. Then, $G^{\prime \prime} \in \Gamma(\pi)$. Since $f(u) \leqslant f\left(w_{j+1}\right)$, by Lemma 5 it follows that $f(v) \leqslant f\left(w_{j+2}\right)$. Let $G^{\prime \prime \prime}=G+u w_{j+2}+v w_{j+3}-u v-w_{j+2} w_{j+3}$. Then, $G^{\prime \prime \prime} \in \Gamma(\pi)$. Since $f(v) \leqslant f\left(w_{j+2}\right), f(u) \leqslant f\left(w_{j+3}\right)$ by Lemma 5 . By repeating the similar arguments, we can conclude that $f\left(w_{s+1}\right) \geqslant \min \{f(u), f(v)\}$, contradicting the condition.

Denote by $\mathcal{R}(G)$ the reduced graph obtained from $G$ by recursively deleting pendant vertices of the resultant graph until no pendant vertices remain. If $G$ is a connected c-cyclic graph, it is easy to see that $\mathcal{R}(G)$ is unique and $\mathcal{R}(G)$ is also a connected $c$-cyclic graph. Thus, we have

$$
\sum_{w \in V(\mathcal{R}(G))} d_{\mathcal{R}(G)}(w)=2|V(\mathcal{R}(G))|+2 c-2 .
$$

Proposition 12. Let $G$ be an extremal graph of $\Gamma(\pi)$, and $f$ be the Perron vector of $G$. Suppose $u \in V(\mathcal{R}(G))$ and $v \in V(G) \backslash V(\mathcal{R}(G))$. Then, $f(u)>f(v)$.

Proof. If $d(v)=1$, then $f(u)>f(v)$ by Corollary 2 and $d(u) \geqslant 2$. Now, we suppose that $d(v) \geqslant 2$. Then, there exists some pendant path, say $P=v x_{1} \cdots x_{s}$, where $d\left(x_{s}\right)=1$.

Suppose $u$ lies on some cycle $C$. Then, there exists some vertex $w \in V(C) \cap N(u)$ such that $w v \notin E(G), w x_{i} \notin E(G)$ and $u x_{i} \notin E(G)$ hold for $1 \leqslant i \leqslant s$. Thus, $f(u)>f(v)$ by Lemma 11. 
Suppose $u$ does not lie on any cycle. Since $u \in \mathcal{R}(G), u$ lies on a path, say $P$, where $P$ is the unique path of $\mathcal{R}(G)$ connecting two cycles, say $C$ and $C^{\prime}$. Suppose $\{x\}=V(P) \cap V(C)$ and $\{y\}=V(P) \cap V\left(C^{\prime}\right)$. Let $P_{v_{1} x}$ be a shortest path connecting $v_{1}$ and $x$, and let $P_{v_{1} y}$ be a shortest path connecting $v_{1}$ and $y$. If $u \in V\left(P_{v_{1} x}\right)$, then $f(u) \geqslant f(x)>f(v)$ by the former arguments and Theorem 10. Similarly, if $u \in V\left(P_{v_{1} y}\right)$, then $f(u) \geqslant f(y)>f(v)$. Now, we consider the case that $u \notin V\left(P_{v_{1} x}\right)$ and $u \notin V\left(P_{v_{1} y}\right)$.

If either $y \in V\left(P_{v_{1} x}\right)$ or $x \in V\left(P_{v_{1} y}\right)$, since $u \notin V\left(P_{v_{1} x}\right)$ and $u \notin V\left(P_{v_{1} y}\right)$, it is easy to see that $u$ lies on a cycle, a contradiction. If $y \notin V\left(P_{v_{1} x}\right)$ and $x \notin V\left(P_{v_{1} y}\right)$, since $u \notin V\left(P_{v_{1} x}\right)$ and $u \notin V\left(P_{v_{1} y}\right), u$ also lies on a cycle, a contradiction.

Proposition 13. Let $G$ be an extremal graph of $\Gamma(\pi)$ and $u$ and $v$ be two vertices of $\mathcal{R}(G)$. Suppose $f$ is the Perron vector of $G$. If $d_{\mathcal{R}(G)}(u)>d_{\mathcal{R}(G)}(v)$, then $f(u)>f(v)$. Furthermore, $f(u)=f(v)$ implies that $d_{\mathcal{R}(G)}(u)=d_{\mathcal{R}(G)}(v)$.

Proof. By Corollary 2, we may suppose that $d_{G}(u) \leqslant d_{G}(v)$. Let $P_{u v}$ be a shortest path from $u$ to $v$ in $\mathcal{R}(G)$. Since $d_{\mathcal{R}(G)}(u)>d_{\mathcal{R}(G)}(v)$, there exists some vertex $w \in N_{\mathcal{R}(G)}(u) \backslash$ $N_{\mathcal{R}(G)}(v)$ so that $w \notin P_{u v}$. Moreover, since $d(u) \leqslant d(v)$ and $d_{\mathcal{R}(G)}(u)>d_{\mathcal{R}(G)}(v)$, there exists some vertex $z$ such that $z \in N_{G}(v) \backslash N_{G}(u)$ and $z \in V(G) \backslash V(\mathcal{R}(G)$ ). By Proposition 12, $f(w)>f(z)$. Let $G^{\prime}=G+u z+v w-u w-v z$. By Corollary $6(1)$, it follows that $f(v)<f(u)$.

Corollary 14. Suppose $G$ is an extremal graph of $\Gamma(\pi)$. If $d_{\mathcal{R}(G)}(v) \geqslant 2$ holds for some $v \in A_{j} \cap \mathcal{R}(G)$ and $j \geqslant 2$, then

(1) $d_{\mathcal{R}(G)}(u) \geqslant d_{\mathcal{R}(G)}(v)$ holds for each $u \in A_{i}$, where $0 \leqslant i \leqslant j-1$;

(2) $d_{\mathcal{R}(G)}(w)=d_{G}(w)$ holds for each $w \in A_{k}$, where $0 \leqslant k \leqslant j-2$.

Proof. (1) clearly follows from Proposition 13 and Theorem 10. Thus, we only need to show (2). Suppose that there exists some vertex $x$ such that $x \in N_{G}(w) \backslash N_{\mathcal{R}(G)}(w)$. Then, $x \notin V(\mathcal{R}(G))$. So, $f(v)>f(x)$ by Proposition 12. On the other hand, since $x \in N_{G}(w)$, $\operatorname{dist}\left(v_{1}, x\right)<\operatorname{dist}\left(v_{1}, v\right)$. By Theorem 10, $f(v) \leqslant f(x)$, a contradiction.

Let $G$ be a connected graph and $T$ be a tree such that $T$ is attached to a vertex $v$ of $G$. Then, $v$ is called the root of $T$. In the coming discussion, we use the notation $T_{v}$ to denote a root tree with root $v$, and we agree that $T_{v}$ includes the root $v$.

An internal path, say $P=v_{1} v_{2} \cdots v_{s+1}(s \geqslant 1)$, is a path joining $v_{1}$ and $v_{s+1}$ (which need not be distinct) such that $v_{1}$ and $v_{s+1}$ have degree greater than 2 , while all other vertices $v_{2}, \ldots, v_{s}$ are of degree 2 . Suppose $P$ is an internal path. Denote $l(P)$ the length of $P$, i.e., $l(P)=s$.

Proposition 15. Let $G$ be an extremal graph of $\Gamma(\pi)$, where $d_{n}=1$. Suppose $P=$ $w_{1} \cdots w_{s+1}$ is an internal path of $\mathcal{R}(G)$.

(1) If $w_{1} \neq w_{s+1}$, then $l(P) \leqslant 2$. Furthermore, if $l(P)=2$, then either $w_{1} w_{3} \in E(G)$ or all the pendant vertices of $G$ are on $T_{w_{2}}$.

(2) If $w_{1}=w_{s+1}$, then $l(P)=3$. 
Proof. Here we only prove (1), since (2) can be demonstrated analogously. Let $f$ be the Perron vector of $G$, and let $f\left(w_{k}\right)=\min \left\{f\left(w_{i}\right)\right.$, where $\left.1 \leqslant i \leqslant s+1\right\}$. Suppose $s \geqslant 3$. By Proposition 13, we have $2 \leqslant k \leqslant s$.

If there exists at least one pendant vertex pertaining to $V(G) \backslash V\left(T_{w_{k}}\right)$, let $G^{\prime}=G+$ $w_{k-1} w_{k+1}+w_{k} w_{k}-w_{k-1} w_{k}-w_{k} w_{k+1}$ (not simple), and let $G^{*}$ be the component of $G^{\prime}$ containing the edge $w_{k-1} w_{k+1}$. Now, Proposition 4 implies that $\rho\left(G^{*}\right) \geqslant \rho(G)$ and $\mu\left(G^{*}\right) \geqslant \mu(G)$. Suppose $u$ is a pendant vertex of $G^{*}$, and $u v \in E\left(G^{*}\right)$. Let $G^{\prime \prime}$ be the graph obtained from $G^{*}$ by subdividing the edge $u v$, i.e., adding a new vertex $w$ and edges $w u, w v$ in $G-u v$. Then, we can construct a new graph $G^{\prime \prime \prime}$ obtained from $G^{\prime \prime}$ (via replacing $T_{w_{k}}$ by $\left.T_{w}\right)$ such that $G^{\prime \prime \prime} \in \Gamma(\pi)$. Since $G^{*} \subset G^{\prime \prime \prime}, \rho\left(G^{\prime \prime \prime}\right)>\rho\left(G^{*}\right) \geqslant \rho(G)$ and $\mu\left(G^{\prime \prime \prime}\right)>\mu\left(G^{*}\right) \geqslant \mu(G)$, a contradiction.

Thus, all the pendant vertices of $G$ are on $T_{w_{k}}$. Since $s \geqslant 3$, there exists some vertex, say $x$, such that $x \in V(P) \backslash\left\{w_{1}, w_{k}, w_{s+1}\right\}, d_{G}\left(w_{k}\right)>2=d_{G}(x)$ and hence $f\left(w_{k}\right)>f(x)$ by Corollary 2, contradicting the choice of $w_{k}$. This contradiction implies that $l(P) \leqslant 2$.

Now, we assume that $w_{1} w_{3} \notin E(G)$ and there exists at least one pendant vertex pertaining to $V(G) \backslash V\left(T_{w_{2}}\right)$. Let $G^{\prime}=G+w_{1} w_{3}+w_{2} w_{2}-w_{1} w_{2}-w_{2} w_{3}$ (not simple). Similarly, we will reach a contradiction, since $f\left(w_{2}\right)<\min \left\{f\left(w_{1}\right), f\left(w_{3}\right)\right\}$ by Proposition 13. So, all the pendant vertices of $G$ are on $T_{w_{2}}$.

By the definition of internal path and Corollary 2, with the similar method as applied in the proof of Proposition 15, we have

Proposition 16. Let $G$ be an extremal graph of $\Gamma(\pi)$, where $d_{n}=1$. Suppose $P$ is an internal path of $G$ from $u$ to $v$. (1) If $u \neq v$, then $l(P) \leqslant 2$ and $u v \in E(G)$. (2) If $u=v$, then $l(P)=3$.

\section{Extremal graphs of $\mathbf{S}(\pi)$}

Denote $P_{n}$ and $K_{n}$, respectively, a path and a complete graph on $n$ vertices. Suppose $u$ is a vertex of $G$, and $P_{s+1}=u_{1} u_{2} \cdots u_{s+1}$, where $u_{i} \notin V(G)$ for $1 \leqslant i \leqslant s+1$. If we obtain $G^{\prime}$ by adding two edges between $u$ and the two pendant vertices of $P_{s+1}$, i.e., by adding the edges $u u_{1}$ and $u u_{s+1}$, then we say that $G^{\prime}$ is obtained from $G$ by appending the path $P_{s+1}$ to $u$ of $G$. If we obtain $G^{\prime}$ by adding the edge $u u_{1}$, then we say that $G^{\prime}$ is obtained from $G$ by attaching the path $P_{s+1}$ to $u$ of $G$.

Suppose $\pi=\left(d_{1}, d_{2}, \ldots, d_{n}\right)$ is a tricyclic degree sequence. Then, $\sum_{i=1}^{n} d_{i}=2 n+4$, which implies that $d_{n} \leqslant 2$ holds for $n \geqslant 5$. It easily follows that

Proposition 17. Suppose $\pi=\left(d_{1}, d_{2}, \ldots, d_{n}\right)$ is a tricyclic degree sequence. If $d_{n}=2$, then $\pi \in\{(6,2, \ldots, 2),(5,3,2, \ldots, 2),(4,4,2, \ldots, 2),(4,3,3,2, \ldots, 2),(3,3,3,3,2, \ldots, 2)\}$. If $d_{n}=1$, then either (1) $d_{1} \geqslant 4$ and $d_{4} \geqslant 3$ or (2) $d_{1}=3$ and $d_{4}=3$ or (3) $d_{2}=d_{3}=$ $d_{4}=2$ or $(4) d_{2}=3$ and $d_{3}=d_{4}=2$ or $(5) d_{2}=d_{3}=3$ and $d_{4}=2$ or $(6) d_{2} \geqslant 4$ and $d_{4}=2$. 
In the following, we shall determined all the extremal tricyclic graphs of $\mathbf{S}(\pi)$ for any prescribed tricyclic degree sequence $\pi$ according to Proposition 17. To do this, we need to introduce more notations as follows.

Let $F_{1}$ be the tricyclic graph obtained by appending two paths of lengths one and a path of length $n-6$, respectively, to a common vertex. Let $D=(V, E)$ be the bicyclic graph such that $V(D)=\left\{u_{1}, u_{2}, u_{3}, u_{4}\right\}$ and $E(D)=\left\{u_{1} u_{2}, u_{1} u_{3}, u_{1} u_{4}, u_{2} u_{3}, u_{2} u_{4}\right\}$. In other words, $D=K_{4}-e$. Let $F_{2}$ be the tricyclic graph obtained from $D$ by appending a path of length $n-5$ to $u_{1}$ of $D$.

Suppose $P_{n-4}=w_{1} w_{2} \cdots w_{n-4}$. Let $F_{3}$ be the tricyclic graph obtained from $D$ and $P_{n-4}$ by adding two edges $u_{1} w_{1}$ and $u_{2} w_{n-4}$. Let $F_{4}$ be the tricyclic graph obtained from $D$ and $P_{n-4}$ by adding two edges $u_{1} w_{1}$ and $u_{3} w_{n-4}$. Let $F_{5}$ be the tricyclic graph obtained from $D$ and $P_{n-4}$ by adding two edges $u_{3} w_{1}$ and $u_{4} w_{n-4}$.

Theorem 18. Suppose $G$ is an extremal of $\mathbf{S}(\pi)$, where $\pi=\left(d_{1}, d_{2}, \ldots, d_{n}\right)$ and $d_{n}=2$.

(1) If $d_{1}=6$ and $d_{2}=\cdots=d_{n}=2$, then $G \cong F_{1}$;

(2) If $d_{1}=5, d_{2}=3$ and $d_{3}=\cdots=d_{n}=2$, then $G \cong F_{2}$;

(3) If $d_{1}=d_{2}=4$ and $d_{3}=\cdots=d_{n}=2$, then $G \cong F_{3}$;

(4) If $d_{1}=4, d_{2}=d_{3}=3$ and $d_{4}=\cdots=d_{n}=2$, then $G \cong F_{4}$;

(5) If $d_{1}=d_{2}=d_{3}=d_{4}=3$ and $d_{5}=\cdots=d_{n}=2$, then $G \cong F_{5}$.

Proof of Theorem $18(1)$. Since $d_{1}=6$ and $d_{2}=\cdots=d_{n}=2, G$ is obtained by appending three paths, say $P_{l_{i}}=w_{i 1} w_{i 2} \cdots w_{i l_{i}}(i=1,2,3)$, respectively, to a common vertex $u$. Without loss of generality, suppose that $l_{1} \geqslant l_{2} \geqslant l_{3}$.

If $l_{2} \geqslant 3$, by Corollary 2 , we have $u=v_{1}$ and $f\left(v_{1}\right)>f\left(v_{2}\right)$. Thus, $f\left(w_{21}\right)>f\left(w_{12}\right)$ and $f\left(w_{11}\right)>f\left(w_{22}\right)$ by Theorem $10(2)$. Let $G^{\prime}=G+w_{11} w_{21}+w_{12} w_{22}-w_{11} w_{12}-w_{21} w_{22}$. By Corollary $6(1), f\left(w_{11}\right)<f\left(w_{22}\right)$, a contradiction.

Therefore, $l_{2}=l_{3}=2$, and hence $G \cong F_{1}$.

Proof of Theorem $18(2)$. By Theorem 10, we can conclude that $v_{1} v_{2} \in E(G)$. If $G$ contains a cut edge, say $u v$, then we may suppose that $u=v_{1}$ and $v=v_{2}$ by Corollary 2 and $d_{3}=2$. Suppose $x \in N\left(v_{1}\right) \backslash\left\{v_{2}\right\}$ and $y \in N\left(v_{2}\right) \backslash\left\{v_{1}\right\}$. By Corollary 2, $f\left(v_{1}\right)>f\left(v_{2}\right)$, and hence $f(x)>f(y)$ by Theorem $10(2)$. Choose $z \in N(x) \backslash\left\{v_{1}\right\}$. Let $G^{\prime}=G+v_{2} x+y z-v_{2} y-x z$. By Corollary $6(1), f\left(v_{2}\right)<f(z)$, a contradiction. Therefore, $G$ contains no cut edge.

Since $d_{3}=2$, there are two paths, say $P_{l_{i}}=v_{1} w_{i 1} w_{i 2} \cdots w_{i l_{i}-2} v_{2}(i=1,2)$, respectively, connecting $v_{1}$ and $v_{2}$ such that $v_{1} v_{2} \notin E\left(P_{l_{1}}\right)$ and $v_{1} v_{2} \notin E\left(P_{l_{2}}\right)$. Without loss of generality, suppose that $l_{1} \geqslant l_{2}$. If $l_{1} \geqslant 4$, choose $x \in N\left(v_{1}\right) \backslash\left\{v_{2}\right\}$ such that $x \notin V\left(P_{l_{1}}\right)$ and $x \notin V\left(P_{l_{2}}\right)$, and let $y \in N(x) \backslash\left\{v_{1}\right\}$. By Corollary 2, $f\left(v_{1}\right)>f\left(v_{2}\right)$, and hence $f(x)>f\left(w_{1 l_{1}-2}\right)$ by Theorem $10(2)$. Let $G^{\prime}=G+v_{2} x+y w_{1 l_{1}-2}-v_{2} w_{1 l_{1}-2}-x y$. Now, Corollary $6(1)$ implies that $f\left(v_{2}\right)<f(y)$, a contradiction. Therefore, $l_{1}=l_{2}=3$, and hence $G \cong F_{2}$. 
Proof of Theorem 18 (3). By Theorem 10, we can conclude that $v_{1} v_{2} \in E(G)$.

Suppose $v_{2}$ is a cut vertex of $G$. Since $d_{3}=2, G$ is obtained from a cycle $C=$ $v_{2} v_{1} w_{11} \cdots w_{1 l_{1}} v_{2}$ by appending the path $P_{l_{2}}=w_{21} w_{22} \cdots w_{2 l_{2}}$ to $v_{1}$ and appending the path $P_{l_{3}}=w_{31} w_{32} \cdots w_{3 l_{3}}$ to $v_{2}$. By Corollary $2, f\left(v_{2}\right)>f\left(w_{22}\right)$, and hence $f\left(w_{21}\right)<$ $f\left(w_{31}\right)$ by Corollary $6(1)$, since $G^{\prime}=G+v_{2} w_{21}+w_{22} w_{31}-w_{21} w_{22}-v_{2} w_{31}$ is connected. On the other hand, since $h\left(w_{31}\right)>h\left(w_{21}\right)$, we have $f\left(w_{21}\right) \geqslant f\left(w_{31}\right)$ by Theorem 10, a contradiction.

Thus, $v_{2}$ is not a cut vertex of $G$. Since $d_{3}=2$, there are three paths, say $P_{l_{i}}=$ $v_{1} w_{i 1} w_{i 2} \cdots w_{i l_{i}-2} v_{2}(i=1,2,3)$, respectively, connecting $v_{1}$ and $v_{2}$ such that $v_{1} v_{2} \notin E\left(P_{l_{i}}\right)$ holds for $i \in\{1,2,3\}$. Without loss of generality, suppose that $l_{1} \geqslant l_{2} \geqslant l_{3}$. Assume that $l_{2} \geqslant 4$. Let $G^{\prime \prime}=G+w_{11} w_{21}+w_{12} w_{22}-w_{11} w_{12}-w_{21} w_{22}$. Then, $G^{\prime \prime}$ is connected.

If $f\left(w_{11}\right)=f\left(w_{22}\right)$ and $f\left(w_{21}\right)=f\left(w_{12}\right), G^{\prime \prime}$ is also an extremal graph of $\mathbf{S}(\pi)$ by Corollary $6(2)$. But $v_{2}$ is a cut vertex of $G^{\prime \prime}$, a contradiction. Thus, either $f\left(w_{11}\right)>f\left(w_{22}\right)$ or $f\left(w_{21}\right)>f\left(w_{12}\right)$ holds by Theorem 10. By Corollary $6(1), f\left(w_{21}\right)>f\left(w_{12}\right)$ implies that $f\left(w_{11}\right)<f\left(w_{22}\right)$ and $f\left(w_{11}\right)>f\left(w_{22}\right)$ implies that $f\left(w_{21}\right)<f\left(w_{12}\right)$, a contradiction.

Thus, $l_{2}=l_{3}=3$, and hence $G \cong F_{3}$.

Proof of Theorem $18(4)$. By Theorem 10, $v_{1} v_{2} \in E(G)$ and $v_{1} v_{3} \in E(G)$.

Case 1. $v_{2} v_{3} \notin E(G)$.

Then, $N\left(v_{1}\right) \cap N\left(v_{2}\right)=\emptyset$ by Theorem $10(1)$.

If $v_{1} v_{2}$ is a cut edge of $G$, choose $x \in N\left(v_{2}\right) \backslash\left\{v_{1}\right\}, y \in N\left(v_{1}\right) \backslash\left\{v_{2}\right\}$ and $z \in$ $N(y) \backslash\left\{v_{1}\right\}$. Since $f\left(v_{1}\right)>f\left(v_{2}\right)$ by Corollary 2, $f(y)>f(x)$ by Theorem 10 (2). Let $G^{\prime}=G+v_{2} y+x z-v_{2} x-y z$. By Corollary $6(1), f\left(v_{2}\right)<f(z)$, a contradiction.

If $v_{1} v_{2}$ is not a cut edge of $G$, choose $x \in N\left(v_{2}\right) \backslash\left\{v_{1}\right\}$ such that $x$ is in a shortest path, say $P$, from $v_{2}$ to $v_{1}$ in $G-v_{1} v_{2}$. Choose $y \in N\left(v_{3}\right) \backslash\left\{v_{1}\right\}$ such that $y$ is not in $P$ (By $d_{4}=2$ and the choice of $P$, such $y$ must exist). Let $G^{\prime}=G+v_{2} v_{3}+x y-v_{2} x-v_{3} y$. Since $f\left(v_{2}\right)>f(y)$ by Corollary 2 , by Corollary $6(1)$, we have $f\left(v_{3}\right)<f(x)$, a contradiction.

Case 2. $v_{2} v_{3} \in E(G)$.

If $v_{1}$ is a cut vertex of $G$, choose $x \in N\left(v_{1}\right) \backslash\left\{v_{2}, v_{3}\right\}$ and $y \in N\left(v_{2}\right) \backslash\left\{v_{1}, v_{3}\right\}$. Let $z$ be a vertex of $N(x) \backslash\left\{v_{1}\right\}$. Since $f\left(v_{1}\right)>f\left(v_{2}\right), f(x)>f(y)$ by Theorem $10(2)$. Let $G^{\prime}=G+v_{2} x+y z-x z-v_{2} y$. By Corollary $6(1)$, we have $f\left(v_{2}\right)<f(z)$, a contradiction.

Thus, $v_{1}$ is not a cut vertex of $G$. Since $d_{4}=2$, there is a path $P_{l_{1}}=v_{1} w_{11} w_{12} \cdots w_{1 l_{1}-2} v_{2}$ connecting $v_{1}$ and $v_{2}$ in $G-v_{3}$, and there is a path $P_{l_{2}}=v_{1} w_{21} w_{22} \cdots w_{2 l_{2}-2} v_{3}$ connecting $v_{1}$ and $v_{3}$ in $G-v_{2}$.

If $l_{1} \geqslant 4$, by Theorem $10(2), f\left(w_{21}\right)>f\left(w_{1 l_{1}-2}\right)$. Let $G^{\prime}=G+w_{21} v_{2}+w_{22} w_{1 l_{1}-2}-$ $w_{21} w_{22}-v_{2} w_{1 l_{1}-2}$ (if $l_{2}=3$, then replace $w_{22}$ by $v_{3}$ ). By Corollary $6(1), f\left(v_{2}\right)<f\left(w_{22}\right)$, a contradiction. Thus, $l_{1}=3$, and hence $G \cong F_{4}$.

Proof of Theorem $18(5)$. By Theorem 10, $v_{1} v_{2} \in E(G), v_{1} v_{3} \in E(G)$ and $v_{1} v_{4} \in E(G)$.

Suppose $G$ contains a cut vertex $u$, where $u \in\left\{v_{2}, v_{3}, v_{4}\right\}$. Without loss of generality, assume that $u=v_{2}$. Choose $x \in N\left(v_{2}\right) \backslash\left\{v_{1}\right\}$ and $y \in N\left(v_{3}\right)$ such that $d(y)=2$. Then, $f\left(v_{2}\right)>f(y)$ by Corollary 2. Let $G^{\prime}=G+v_{2} v_{3}+x y-v_{2} x-v_{3} y$. By Corollary $6(1)$, $f\left(v_{3}\right)<f(x)$, which contradicts $d\left(v_{3}\right)>d(x)=2$ and Corollary 2. 
Thus, $G$ is obtained from a cycle $C=u w_{11} \cdots w_{1 l_{1}} v w_{21} \cdots w_{2 l_{2}} w w_{31} \cdots w_{3 l_{3}} u$ and an isolated vertex $z$ by adding three edges $z u, z v$, and $z w$. Without loss of generality, suppose that $l_{1} \geqslant l_{2} \geqslant l_{3} \geqslant 0$. If $l_{2} \geqslant 1$, then $f(u)>f\left(w_{21}\right)$ and $f(v)>f\left(w_{11}\right)$ by Corollary 2. Let $G^{\prime}=G+u v+w_{11} w_{21}-u w_{11}-v w_{21}$. By Corollary $6(1), f(v)<f\left(w_{11}\right)$, a contradiction.

Therefore, $l_{2}=l_{3}=0$, and hence $G \cong F_{5}$.

Lemma 19. Suppose $G$ is an extremal of $\mathbf{S}(\pi)$, and $v \in \mathcal{R}(G)$ such that $\operatorname{dist}\left(v_{1}, v\right)$ is as large as possible. If $v \notin A_{1}$ and $d_{n}=1$, then $v \in A_{2}, d_{\mathcal{R}(G)}(v)=2$, and the two neighbors of $v$ of $\mathcal{R}(G)$ pertain to $A_{1}$.

Proof. If $d_{\mathcal{R}(G)}(v) \geqslant 3$ holds for some $v \in A_{j} \cap \mathcal{R}(G)$ and $j \geqslant 2$, by Corollary 14 (1), $d_{\mathcal{R}(G)}(u) \geqslant 3$ holds for every $u \in A_{i}$, where $0 \leqslant i \leqslant j-1$. Thus, $\sum_{w \in V(\mathcal{R}(G))} d_{\mathcal{R}(G)}(w) \geqslant$ $5 \times 3+2(|V(\mathcal{R}(G))|-5)>2|V(\mathcal{R}(G))|+4$, contradicting equation $(1)$. So, $d_{\mathcal{R}(G)}\left(v^{\prime}\right)=2$ holds for each $v^{\prime} \in A_{j} \cap \mathcal{R}(G)$, where $j \geqslant 2$.

Suppose that there exists some $v \in A_{j} \cap \mathcal{R}(G)$, where $j \geqslant 3$. Since $d_{\mathcal{R}(G)}\left(v^{\prime}\right)=2$ holds for each $v^{\prime} \in A_{2} \cap \mathcal{R}(G)$, by Corollary 14, $v$ lies on an internal path $P$ of $\mathcal{R}(G)$ such that $l(P) \geqslant 4$, contradicting Proposition 15. Therefore, $v \in A_{2}$ and $d_{\mathcal{R}(G)}(v)=2$.

To complete the proof, it suffices to show the following claim.

Claim. If $v \in A_{2} \cap V(\mathcal{R}(G))$, then the two neighbors of $v$ of $\mathcal{R}(G)$ pertain to $A_{1}$.

Assume the claim is not true, then at least one of the two neighbors of $v$ of $\mathcal{R}(G)$ does not belong to $A_{1}$. We may assume that $w$ is such a neighbor of $v$. Then, $d_{\mathcal{R}(G)}(w)=2$ and $w \in A_{2} \cap V(\mathcal{R}(G))$ by the former arguments. We consider the following two cases.

Case 1. $v$ and $w$ do not lie on a triangle.

Then, $v$ lies on an internal path $P$ from $x$ to $y$, where $\{x, y\} \subseteq V(\mathcal{R}(G))$ by Corollary 14. Furthermore, $x=y$ implies that $l(P) \geqslant 4$ and $x \neq y$ implies that $l(P) \geqslant 3$, which is a contradiction to Proposition 15.

Case 2. $v$ and $w$ lie on a triangle, say $C$, where $V(C)=\{u, v, w\}$.

By Corollary 14, $A_{1} \cap N_{\mathcal{R}(G)}(v)=\{u\}=A_{1} \cap N_{\mathcal{R}(G)}(w)$.

Subcase 2.1. There exists some vertex $x$ such that $x \in A_{2} \cap(V(\mathcal{R}(G)) \backslash\{v, w\})$.

By Case 1, either there exist vertices $y \in A_{1} \cap V(\mathcal{R}(G))$ and $z \in A_{2} \cap V(\mathcal{R}(G))$ such that $x, y, z$ form a triangle, or there exist vertices $y, z \in A_{1} \cap V(\mathcal{R}(G))$ such that $N_{\mathcal{R}(G)}(x)=\{y, z\}$.

We first suppose that there exist vertices $y \in A_{1} \cap V(\mathcal{R}(G))$ and $z \in A_{2} \cap V(\mathcal{R}(G))$ such that $x, y, z$ form a triangle. If $u=y$, then $d_{\mathcal{R}(G)}\left(v_{1}\right) \geqslant d_{\mathcal{R}(G)}(u) \geqslant 5$ by Proposition 13 , and hence $\sum_{w \in V(\mathcal{R}(G))} d_{\mathcal{R}(G)}(w) \geqslant 5 \times 2+2(|V(\mathcal{R}(G))|-2)>2|V(\mathcal{R}(G))|+4$, contradicting equation (1). If $u y \in E(G)$, then $d_{\mathcal{R}(G)}\left(v_{1}\right)=2<d_{G}\left(v_{1}\right)$, contradicting Corollary 14 (2).

Thus, $u \neq y$ and $u y \notin E(G)$. By Proposition 13, $f(u)>f(x)$ and $f(y)>f(v)$. Let $G^{\prime}=G+u y+v x-u v-y x$. Corollary $6(1)$ implies that $f(y)<f(v)$, a contradiction.

Now, we suppose that there exist vertices $y, z \in A_{1} \cap V(\mathcal{R}(G))$ such that $N_{\mathcal{R}(G)}(x)=$ $\{y, z\}$.

If $u=y$, by Proposition $13, d_{\mathcal{R}(G)}\left(v_{1}\right) \geqslant d_{\mathcal{R}(G)}(u) \geqslant 4$. We claim that $d_{\mathcal{R}(G)}(z) \geqslant 3$. Otherwise, $P=y x z v_{1}$ is an internal path of $\mathcal{R}(G)$ of length three, contradicting Proposition 15 (1). So, $d_{\mathcal{R}(G)}(z) \geqslant 3$. On the other hand, recall that $d_{\mathcal{R}(G)}\left(v_{1}\right) \geqslant d_{\mathcal{R}(G)}(u) \geqslant 4$, it is a contradiction to equation (1). Thus, $u \neq y$. Similarly, $u \neq z$. 
Since $G$ is a tricyclic graph, either uy $\notin E(G)$ or $u z \notin E(G)$. We may suppose that $u z \notin E(G)$. By Proposition 13 and Theorem 10, $f(u)>f(x)$ and $f(z) \geqslant f(v)$. Let $G^{\prime}=G+u z+v x-u v-z x$. Now, Corollary 6 (1) implies that $f(z)<f(v)$, a contradiction.

Subcase 2.2. $A_{2} \cap V(\mathcal{R}(G))=\{v, w\}$.

If $u x \in E(G)$ holds for every $x \in N\left(v_{1}\right) \backslash\{u\}$, by Proposition 13 , we have $d_{\mathcal{R}(G)}\left(v_{1}\right) \geqslant$ $d_{\mathcal{R}(G)}(u) \geqslant 5$, contradicting equation (1). Thus, there exists at least one vertex, say $y$, of $N\left(v_{1}\right) \backslash\{u\}$ such that $u y \notin E(G)$, and there exists a vertex $z$ in $N\left(v_{1}\right) \backslash\{u, y\}$ such that $y z \in E(G)$.

If $u z \in E(G)$, by Proposition 13 , we have $d_{\mathcal{R}(G)}\left(v_{1}\right) \geqslant d_{\mathcal{R}(G)}(u) \geqslant 4$ and $d_{\mathcal{R}(G)}(z) \geqslant 3$, contradicting equation (1). Thus, $u z \notin E(G)$ and $u y \notin E(G)$.

Since $G$ is a tricyclic graph, either $d_{\mathcal{R}(G)}(y)=2$ or $d_{\mathcal{R}(G)}(z)=2$. We may suppose that $d_{\mathcal{R}(G)}(z)=2$. By Proposition 13 and Theorem 10, $f(u)>f(z)$ and $f(y) \geqslant f(v)$. Let $G^{\prime}=G+u y+v z-u v-y z$. Now, Corollary 6 (1) implies that $f(y)<f(v)$, a contradiction.

Lemma 20. Suppose $G$ is an extremal of $\mathbf{S}(\pi)$, and $v \in \mathcal{R}(G)$ such that $\operatorname{dist}\left(v_{1}, v\right)$ is as large as possible. If $d_{1} \geqslant 4$ and $d_{n}=1$, then $v \in A_{1}$.

Proof. Suppose $v \notin A_{1}$. By Lemma 19, $v \in A_{2}$ with $d_{\mathcal{R}(G)}(v)=2$, and we may suppose that $x$ and $y$ are the two neighbors of $v$ in $\mathcal{R}(G) \cap A_{1}$. Furthermore, Corollary 14 (1) implies that $w \in \mathcal{R}(G)$ holds for each $w \in A_{1}$.

If $x y \notin E(G)$, by Proposition 15 (1), all the pendant vertices of $G$ lie on $T_{v}$. So, $d_{G}(v) \geqslant 3$. By Corollary $2, d_{\mathcal{R}(G)}(w)=d_{G}(w) \geqslant d_{G}(v) \geqslant 3$ holds for each $w \in A_{1} \cup\left\{v_{1}\right\}$, since $f(w) \geqslant f(v)$ by Theorem 10. Thus, $\sum_{w \in V(\mathcal{R}(G))} d_{\mathcal{R}(G)}(w) \geqslant 4+4 \times 3+2(|V(\mathcal{R}(G))|-$ 5) $>2|V(\mathcal{R}(G))|+4$, which contradicts equation (1).

If $x y \in E(G)$, then $d_{1}=4$ and $d_{\mathcal{R}(G)}\left(v_{4}\right)=d_{\mathcal{R}(G)}\left(v_{5}\right)=2$ by equation (1) and Corollary 14 (2). By the virtue of the former arguments and equation (1), we may suppose that $A_{2} \cap V(\mathcal{R}(G))=\{v\}$ and hence $v_{4} v_{5} \in E(G)$. By Proposition 13 and Theorem 10, we have $f\left(v_{2}\right)>f\left(v_{5}\right)$ and $f\left(v_{4}\right) \geqslant f(v)$. Let $G^{\prime}=G+v_{2} v_{4}+v_{5} v-v_{4} v_{5}-v_{2} v$. By Corollary $6(1), f\left(v_{4}\right)<f(v)$, a contradiction.

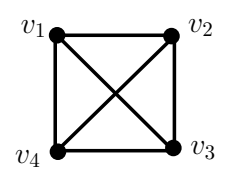

$H_{1}$

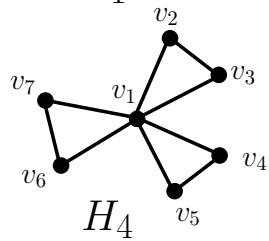

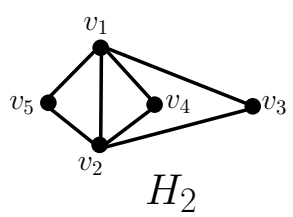

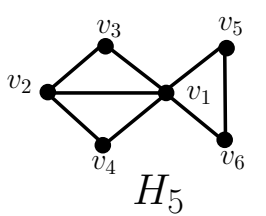

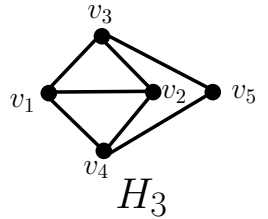

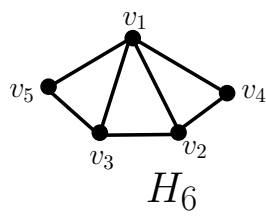

Figure 1: The tricyclic graphs $H_{1}, H_{2}, \ldots, H_{6}$. 
In the following, let $H_{1}, H_{2}, \ldots, H_{6}$ be the tricyclic graphs as shown in Figure 1.

Lemma 21. Suppose $G$ is an extremal of $\mathbf{S}(\pi)$, and $v \in \mathcal{R}(G)$ such that $\operatorname{dist}\left(v_{1}, v\right)$ is as large as possible. If $v \in A_{1}$, then $\mathcal{R}(G) \in\left\{H_{1}, H_{2}, H_{4}, H_{5}, H_{6}\right\}$.

Proof. Since $\mathcal{R}(G)$ is also a tricyclic graph, $1 \leqslant \max \left\{\left|N_{\mathcal{R}(G)}(u) \cap N_{\mathcal{R}(G)}\left(v_{1}\right)\right|: u \in\right.$ $\left.V(\mathcal{R}(G)) \backslash\left\{v_{1}\right\}\right\} \leqslant 3$. If $\max \left\{\left|N_{\mathcal{R}(G)}(u) \cap N_{\mathcal{R}(G)}\left(v_{1}\right)\right|: u \in V(\mathcal{R}(G)) \backslash\left\{v_{1}\right\}\right\}=1$, then $\mathcal{R}(G) \cong H_{4}$ by Proposition 12-13 and Lemma 9. If $\max \left\{\left|N_{\mathcal{R}(G)}(u) \cap N_{\mathcal{R}(G)}\left(v_{1}\right)\right|: u \in\right.$ $\left.V(\mathcal{R}(G)) \backslash\left\{v_{1}\right\}\right\}=2$, then $\mathcal{R}(G) \cong H_{1}$ or $\mathcal{R}(G) \cong H_{5}$ or $\mathcal{R}(G) \cong H_{6}$ by Theorem 10 (1) and Propositions 12-13. Similarly, if $\max \left\{\left|N_{\mathcal{R}(G)}(u) \cap N_{\mathcal{R}(G)}\left(v_{1}\right)\right|: u \in V(\mathcal{R}(G)) \backslash\left\{v_{1}\right\}\right\}=$ 3 , then $\mathcal{R}(G) \cong H_{2}$.

Lemma 22. Suppose $G$ is an extremal of $\mathbf{S}(\pi)$, where $\pi=\left(d_{1}, d_{2}, \ldots, d_{n}\right)$ and $d_{n}=1$.

(1) If $d_{2} \geqslant 3$, then $\mathcal{R}(G) \approx H_{4}$;

(2) If $d_{2} \geqslant 4$ or $d_{3} \geqslant 3$, then $\mathcal{R}(G) \neq H_{5}$;

(3) If $d_{2} \geqslant 4$ or $d_{4} \geqslant 3$, then $\mathcal{R}(G) \neq H_{6}$.

Proof. (1) Assume that $\mathcal{R}(G) \cong H_{4}$. Since $d_{2} \geqslant 3$, there exists some neighbor, say $x$, of $v_{2}$ such that $x \in V(G) \backslash V(\mathcal{R}(G))$. By Lemma 11, we have $f\left(v_{6}\right)>f\left(v_{2}\right)$, a contradiction.

(2) Assume that $\mathcal{R}(G) \cong H_{5}$. If $d_{3} \geqslant 3$, there exists some neighbor, say $x$, of $v_{3}$ such that $x \in V(G) \backslash V(\mathcal{R}(G))$. By Lemma 11, we have $f\left(v_{6}\right)>f\left(v_{3}\right)$, a contradiction. If $d_{2} \geqslant 4$, we will yield a similar contradiction.

(3) Assume that $\mathcal{R}(G) \cong H_{6}$. If $d_{4} \geqslant 3$, by Lemma 11 we have $f\left(v_{5}\right)>f\left(v_{4}\right)$, a contradiction. If $d_{2} \geqslant 4$, by Lemma 11 , we have $f\left(v_{3}\right)>f\left(v_{2}\right)$, a contradiction.

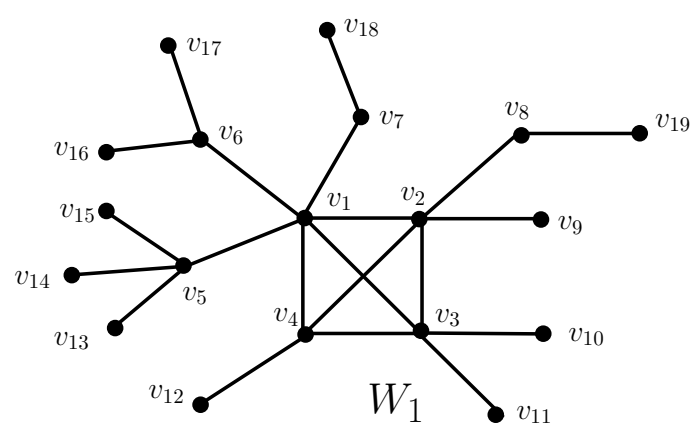

Figure 2: The tricyclic graph $W_{1}$.

Let $W_{1}$ be the unique tricyclic graph with $\mathcal{R}\left(W_{1}\right)=H_{1}$, and the remaining vertices appear in $B F S$-ordering (also called spiral like dispositions in $[1,11]$ ) with respect to $H_{1}$ starting from $v_{5}$ that is adjacent to $v_{1}$. It means that, $W_{1}$ can be constructed by the breadth-first-search method as follows: Select a vertex $v_{1}$ as a root and begin with $v_{1}$ of 
the zeroth layer. Select the vertices $v_{2}, \ldots, v_{d_{1}+1}$ as the first layer such that $v_{2}, \ldots, v_{d_{1}+1}$ are adjacent with $v_{1}$. Let

$$
\begin{aligned}
& N\left(v_{2}\right)=\left\{v_{1}, v_{3}, v_{4}, v_{d_{1}+2}, v_{d_{1}+3}, \ldots, v_{d_{1}+d_{2}-2}\right\}, \\
& N\left(v_{3}\right)=\left\{v_{1}, v_{2}, v_{4}, v_{d_{1}+d_{2}-1}, \ldots, v_{d_{1}+d_{2}+d_{3}-5}\right\}, \\
& N\left(v_{4}\right)=\left\{v_{1}, v_{2}, v_{3}, v_{d_{1}+d_{2}+d_{3}-4}, \ldots, v_{d_{1}+d_{2}+d_{3}+d_{4}-8}\right\}, \text { and } \\
& N\left(v_{5}\right)=\left\{v_{1}, v_{d_{1}+d_{2}+d_{3}+d_{4}-7}, \ldots, v_{d_{1}+d_{2}+d_{3}+d_{4}+d_{5}-9}\right\}, \text { etc. }
\end{aligned}
$$

Informally, for a given tricyclic degree sequence $\pi=\left(6,5^{(2)}, 4^{(2)}, 3,2^{(2)}, 1^{(11)}\right), W_{1}$ is the tricyclic graph of order 19 as shown in Figure 2.

Let $W_{2}$ (respectively, $W_{5}, W_{6}$ ) be the unique tricyclic graph with $\mathcal{R}\left(W_{2}\right)=H_{2}$ (respectively, $\left.\mathcal{R}\left(W_{5}\right)=H_{5}, \mathcal{R}\left(W_{6}\right)=H_{6}\right)$, and the remaining vertices appear in $B F S$-ordering with respect to $H_{2}$ (respectively, $H_{5}, H_{6}$ ) starting from $v_{6}$ (respectively, $v_{7}, v_{6}$ ) that is adjacent to $v_{1}$. Denote $W_{3}$ the unique tricyclic graph with $\mathcal{R}\left(W_{3}\right)=H_{3}$ so that the remaining vertices appear in $B F S$-ordering with respect to $H_{3}$ starting from $v_{6}$ that is adjacent to $v_{5}$.

Paths $P_{l_{1}}, \ldots, P_{l_{k}}$ are said to have almost equal lengths if $l_{1}, \ldots, l_{k}$ satisfy $\left|l_{i}-l_{j}\right| \leqslant 1$ for $1 \leqslant i \leqslant j \leqslant k$. Let $W_{4}$ be the unique tricyclic graph obtained from $H_{4}$ by attaching $k$ paths of almost equal lengths to $v_{1}$ of $H_{4}$, where $d_{1}=k+6$.

Theorem 23. Suppose $G$ is an extremal of $\mathbf{S}(\pi)$, where $\pi=\left(d_{1}, d_{2}, \ldots, d_{n}\right)$ and $d_{n}=1$.

(1) If $d_{1} \geqslant 4, d_{4} \geqslant 3$, then $G \cong W_{1}$ or $G \cong W_{2}$;

(2) If $d_{1}=3$ and $d_{4}=3$, then $G \cong W_{3}$;

(3) If $d_{2}=d_{3}=d_{4}=2$, then $G \cong W_{4}$;

(4) If $d_{2}=3$ and $d_{3}=d_{4}=2$, then $G \cong W_{5}$;

(5) If $d_{2}=d_{3}=3$ and $d_{4}=2$, then $G \cong W_{6}$;

(6) If $d_{2} \geqslant 4$ and $d_{4}=2$, then $G \cong W_{2}$.

Proof. Choose $v \in \mathcal{R}(G)$ such that $\operatorname{dist}\left(v_{1}, v\right)$ is as large as possible.

(1) By Lemma 20, $v v_{1} \in E(G)$. By Lemmas $21-22$, we can conclude that $\mathcal{R}(G) \cong H_{1}$ or $\mathcal{R}(G) \cong H_{2}$. Thus, either $G \cong W_{1}$ or $G \cong W_{2}$ by Theorem 10 .

(3) Since $G$ is a tricyclic graph, $d_{1} \geqslant 7$. Otherwise, $\sum_{i=1}^{n} d_{i} \leqslant 6+2(n-2)+1=2 n+3$, a contradiction. By Lemmas 20-21 and Theorem 10, we have $G \cong W_{4}$.

(4) Since $G$ is a tricyclic graph, $d_{1} \geqslant 6$. Otherwise, $\sum_{i=1}^{n} d_{i} \leqslant 5+3+2(n-3)+1=$ $2 n+3$, a contradiction. By Lemmas $20-22, \mathcal{R}(G) \cong H_{5}$, and hence $G \cong W_{5}$ by Theorem 10.

(5) Since $G$ is a tricyclic graph, $d_{1} \geqslant 4$. Otherwise, $\sum_{i=1}^{n} d_{i} \leqslant 3 \times 3+2(n-4)+1=$ $2 n+2$, a contradiction. By Lemmas 20-22 and Theorem 10, we have $G \cong W_{6}$.

(6) By Lemma 20, $v v_{1} \in E(G)$, and hence $\mathcal{R}(G) \cong H_{2}$ by Lemmas 21-22. Now, Theorem 10 implies that $G \cong W_{2}$. 
(2) Since $G$ is a tricyclic graph, $d_{1}=d_{2}=\cdots=d_{5}=3$ and $d_{n}=1$. By Lemma 19 , either $v \in A_{2}$ with $d_{\mathcal{R}(G)}(v)=2$ or $v v_{1} \in E(G)$. If $v v_{1} \in E(G)$, then $\mathcal{R}(G) \cong H_{1}$ by Lemmas 21-22 and hence $G$ is not connected, a contradiction. Thus, $v \in A_{2}$ with $d_{\mathcal{R}(G)}(v)=2$, and hence $d_{\mathcal{R}(G)}\left(v_{1}\right)=3$ and $d_{\mathcal{R}(G)}\left(v_{2}\right) \geqslant d_{\mathcal{R}(G)}\left(v_{3}\right) \geqslant d_{\mathcal{R}(G)}\left(v_{4}\right) \geqslant 2$ by Corollary 14.

By Lemma 19, let $x$ and $y$ be the two neighbors of $v$ of $\mathcal{R}(G)$ in $A_{1}$.

If $x y \in E(G)$, then $d_{\mathcal{R}(G)}(z)=3$, where $z \in\left\{v_{2}, v_{3}, v_{4}\right\} \backslash\{x, y\}$ and there exist two vertices, say $u$ and $w$, such that $u, w \in A_{2}$ and $z, u, w$ form a triangle, which contradicts Lemma 19.

If $x y \notin E(G)$, by Proposition 15 (1), all the pendant vertices of $G$ are on $T_{v}$. So, $d_{G}(v)=3$. By Corollary 2, we can conclude that $d_{\mathcal{R}(G)}\left(v_{2}\right)=d_{\mathcal{R}(G)}\left(v_{3}\right)=d_{\mathcal{R}(G)}\left(v_{4}\right)=3$. Thus, $G \cong W_{3}$ by Theorem 10, Lemma 19 and Proposition 15 (1).

\section{Further discussion}

In view of Theorem 23, it is natural to consider the following question: Whether the construction of $G$ of Theorem 23 (1) is unique? Unfortunately, as the following example shown, the answer is negative.

Example 24. Suppose $p \geqslant q \geqslant 0$ are two integers. Let $S_{1}$ and $S_{2}$ be the tricyclic graphs as shown in Figure 3. Let $S_{1}(p, q)$ (respectively, $S_{2}(p, q)$ ) be the tricyclic graph obtained from $S_{1}$ (respectively, $S_{2}$ ) by attaching $p$ pendant vertices to $v_{1}$, and attaching $q$ pendant vertices to $v_{2}$. Let $G$ be the extremal graph of $\mathbf{S}(\pi)$, where $\pi=\left(p+4, q+4,4^{(2)}, 3,1^{(p+q+5)}\right)$. Theorem 23 (1) implies that either $G \cong S_{1}(p, q)$ or $G \cong S_{2}(p, q)$. Using "Matlab", it easily follows that $\rho\left(S_{1}(4,2)\right)>3.7363>3.6888>\rho\left(S_{2}(4,2)\right), \rho\left(S_{1}(15,10)\right)<4.9168<$ $4.9238<\rho\left(S_{2}(15,10)\right), \mu\left(S_{1}(4,2)\right)<9.7373<9.7374<\mu\left(S_{2}(4,2)\right)$, and $\mu\left(S_{1}(1,1)\right)>$ $7.8243>7.7439>\mu\left(S_{2}(1,1)\right)$.
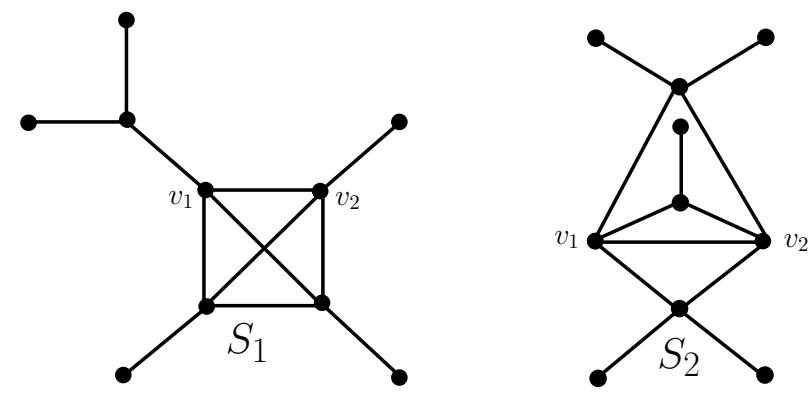

Figure 3: The tricyclic graphs $S_{1}$ and $S_{2}$.

Now, we present our main result of this section as follows.

Proposition 25. Let $\pi=\left(d_{1}, d_{2}, \ldots, d_{n}\right)$ and $\pi^{\prime}=\left(d_{1}^{\prime}, d_{2}^{\prime}, \ldots, d_{n}^{\prime}\right)$ be two different tricyclic degree sequences, and $\pi \triangleleft \pi^{\prime}$. Suppose $G$ and $G^{\prime}$ are the extremal graphs of $\mathbf{S}(\pi)$ and $\mathbf{S}\left(\pi^{\prime}\right)$, respectively. 
(1) If $d_{n} \geqslant 2$, then $\rho(G)<\rho\left(G^{\prime}\right)$ and $\mu(G)<\mu\left(G^{\prime}\right)$;

(2) If $d_{i}=d_{i}^{\prime}$ holds for $1 \leqslant i \leqslant 4$, then $\rho(G)<\rho\left(G^{\prime}\right)$ and $\mu(G)<\mu\left(G^{\prime}\right)$;

(3) Suppose there exists some $t$ such that $d_{t}^{\prime} \geqslant 3$ and $d_{i}=d_{i}^{\prime}$ holds for all $1+t \leqslant i \leqslant n$. If $d_{1}=d_{1}^{\prime}$, then $\rho(G)<\rho\left(G^{\prime}\right)$ and $\mu(G)<\mu\left(G^{\prime}\right)$;

(4) Suppose there exists some $t$ such that $d_{t}^{\prime} \geqslant 2$ and $d_{i}=d_{i}^{\prime}$ holds for all $1+t \leqslant i \leqslant n$. If $d_{1}=d_{1}^{\prime}, d_{2}=d_{2}^{\prime}$ and $d_{3}=d_{3}^{\prime}$, then $\rho(G)<\rho\left(G^{\prime}\right)$ and $\mu(G)<\mu\left(G^{\prime}\right)$.

To prove Proposition 25, we need to introduce more lemmas as follows.

Denote $\Phi(G, x)$ the characteristic polynomial of the adjacency matrix of $G$. The following result is often used to calculate $\Phi(G, x)$ of a graph $G$.

Lemma 26. [14] (Schwenk's formulas) Let $G$ be a graph. Denote by $C_{v}$ the set of all cycles in $G$ containing a vertex $v$. Then,

$$
\Phi(G, x)=x \Phi(G-v, x)-\sum_{w \sim v} \Phi(G-v-w, x)-2 \sum_{C \in C_{v}} \Phi(G-V(C), x) .
$$

Lemma 27. If $n \geqslant 7$, then $\rho\left(F_{1}\right)>\rho\left(F_{2}\right)$ and $\mu\left(F_{1}\right)>\mu\left(F_{2}\right)$.

Proof. We first show that $\rho\left(F_{1}\right)>\rho\left(F_{2}\right)$. Applying Lemma 26 to $v_{1}$ of $F_{1}$ and $F_{2}$, respectively, it follows that

$$
\begin{aligned}
& \Phi\left(F_{1}, x\right)=(x-1)\left(x^{2}-x-4\right)(x+1)^{2} \Phi\left(P_{n-5}, x\right)-2\left(x^{2}-1\right)^{2} \Phi\left(P_{n-6}, x\right)-2\left(x^{2}-1\right)^{2} \\
& \Phi\left(F_{2}, x\right)=x(x+1)\left(x^{2}-x-4\right) \Phi\left(P_{n-4}, x\right)-2 x\left(x^{2}-2\right) \Phi\left(P_{n-5}, x\right)-2 x\left(x^{2}-2\right)
\end{aligned}
$$

Furthermore, Lemma 26 implies that $\Phi\left(P_{n}, x\right)=x \Phi\left(P_{n-1}, x\right)-\Phi\left(P_{n-2}, x\right)$. Thus, by equations (2) and (3), we have

$\Phi\left(F_{2}, x\right)-\Phi\left(F_{1}, x\right)=2 \Phi\left(P_{n-6}, x\right)+\left(x^{3}+x+4\right) \Phi\left(P_{n-7}, x\right)+2\left(x^{4}-x^{3}-2 x^{2}+2 x+1\right)$.

Note that $x^{4}-x^{3}-2 x^{2}+2 x+1>0$ when $x \geqslant \rho\left(F_{1}\right)>\sqrt{d_{F_{1}}\left(v_{1}\right)}=\sqrt{6}$. Thus, when $x \geqslant \rho\left(F_{1}\right)>2>\rho\left(P_{n-6}\right), \Phi\left(F_{2}, x\right)>\Phi\left(F_{1}, x\right)$, which implies that $\rho\left(F_{1}\right)>\rho\left(F_{2}\right)$.

Now we turn to verify that $\mu\left(F_{1}\right)>\mu\left(F_{2}\right)$. Since $F_{1}$ is not bipartite, then $\mu\left(F_{1}\right)>$ $d_{F_{1}}\left(v_{1}\right)+1=7$ (see e. g. [13]). By the upper bound of $[7]$ for $\mu(G)$, we have

$$
\mu\left(F_{2}\right) \leqslant \max \left\{\frac{d(u)(d(u)+m(u))+d(v)(d(v)+m(v))}{d(u)+d(v)}, u v \in E\left(F_{2}\right)\right\} \leqslant \frac{48}{7}<\mu\left(F_{1}\right),
$$

where $m(v)=\sum_{w \in N(v)} d(w) / d(v)$. So, the result follows.

If $d=\left(d_{1}, \ldots, d_{n}\right)$ is a non-increasing integer sequence and $d_{i} \geqslant d_{j}+2$, then the following operation is called a unit transformation from $i$ to $j$ on $d$ : subtract 1 from $d_{i}$ and add 1 to $d_{j}$. The following famous lemma on majorization of integer sequences, is due to Muirhead (see [12]). 
Lemma 28. (Muirhead's Lemma) If $d$ and $d^{\prime}$ are two non-increasing integer sequences and $d \triangleleft d^{\prime}$, then $d$ can be obtained from $d^{\prime}$ by a finite sequence of unit transformations.

Suppose $\pi \triangleleft \pi^{\prime}, G$ and $G^{\prime}$ are the extremal graphs of $\Gamma(\pi)$ and $\Gamma\left(\pi^{\prime}\right)$, respectively. In the following, by Lemma 28 , we may always suppose that $\pi$ and $\pi^{\prime}$ differ only in two positions where the difference is 1 , that is, $d_{i}=d_{i}^{\prime}, i \neq p, q, 1 \leqslant p<q \leqslant n$, and $d_{p}^{\prime}=d_{p}+1$, $d_{q}^{\prime}=d_{q}-1$. Let $f$ be the Perron vector of $G$, and let $P_{v_{p} v_{q}}$ be a shortest path from $v_{p}$ to $v_{q}$. By the choice of $G$ and $p<q, f\left(v_{p}\right) \geqslant f\left(v_{q}\right)$ follows from Theorem 10. In the following, if $w$ is a vertex of $G$ such that $w \in N\left(v_{q}\right) \backslash\left(N\left(v_{p}\right) \cup\left\{v_{p}\right\}\right)$ and $w \notin V\left(P_{v_{p} v_{q}}\right)$, then we call $w$ a surprising vertex of $G$. If $G$ contains some surprising vertex, say $w$, let $G^{*}=G+v_{p} w-v_{q} w$. Then, $G^{*} \in \Gamma\left(\pi^{\prime}\right)$. Since $f\left(v_{p}\right) \geqslant f\left(v_{q}\right)$, Lemma 1 implies that $\rho(G)<\rho\left(G^{*}\right) \leqslant \rho\left(G^{\prime}\right)$ and $\mu(G)<\mu\left(G^{*}\right) \leqslant \mu\left(G^{\prime}\right)$. Therefore, if $G$ contains a surprising vertex, then $\rho(G)<\rho\left(G^{\prime}\right)$ and $\mu(G)<\mu\left(G^{\prime}\right)$.

Proof of Proposition 25. It is easy to check that the result follows for $n \leqslant 6$ with the aid of computer. Thus, we may suppose that $n \geqslant 7$ in the following.

(1) If $\pi=(5,3,2, \ldots, 2)$ and $\pi^{\prime}=(6,2,2, \ldots, 2)$, then $G=F_{2}$ and $G^{\prime}=F_{1}$ by Theorem 18. Now, the result follows from Lemmas 27. If $\pi=(3,3,3,3,2, \ldots, 2)$, then $\pi^{\prime}=(4,3,3,2, \ldots, 2)$. By Theorem $18, G=F_{5}$ and $G^{\prime}=F_{4}$. Without loss of generality, suppose $f\left(u_{3}\right) \geqslant f\left(u_{4}\right)$. Choose $x \in N\left(u_{4}\right) \backslash\left\{u_{1}, u_{2}\right\}$. Then, $x$ is a surprising vertex of $F_{5}$, and hence $\rho\left(F_{5}\right)<\rho\left(F_{4}\right)$ and $\mu\left(F_{5}\right)<\mu\left(F_{4}\right)$. We can also employ the similar method to deal with the other cases by Theorems 10 and 18 .

(2) Since $d_{i}=d_{i}^{\prime}$ holds for $1 \leqslant i \leqslant 4, q>p \geqslant 5$ and $d_{n}=1$ by Theorem 18 .

If $q \geqslant 8$ or $d_{7} \geqslant 3$, by Theorem $23 G$ contains some surprising vertex.

If $q=7$ and $d_{7}=2$, then $p=6$ or $p=5$. When $p=5, G$ contains some surprising vertex according to Theorem 23. When $p=6$, then $d_{5}=d_{5}^{\prime} \geqslant d_{6}^{\prime}=d_{6}+1 \geqslant 3$. By Theorem 23, $G$ contains some surprising vertex.

If $q=6$, then $p=5$, and hence $d_{4}=d_{4}^{\prime} \geqslant d_{5}^{\prime}=d_{5}+1 \geqslant 3$. By Theorem 23, $G$ contains some surprising vertex.

(3) Note that $d_{t}^{\prime} \geqslant 3$ and $d_{i}=d_{i}^{\prime}$ holds for all $1+t \leqslant i \leqslant n$. Then, $d_{q}=d_{q}^{\prime}+1 \geqslant$ $d_{t}^{\prime}+1 \geqslant 4$. Since $d_{1}=d_{1}^{\prime}, G$ contains some surprising vertex according to Theorem 18 and Theorem 23.

(4) Note that $d_{t}^{\prime} \geqslant 2$ and $d_{i}=d_{i}^{\prime}$ holds for all $1+t \leqslant i \leqslant n$. Then, $d_{q}=d_{q}^{\prime}+1 \geqslant$ $d_{t}^{\prime}+1 \geqslant 3$ and $d_{p}^{\prime}=d_{p}+1 \geqslant d_{q}+1 \geqslant 4$. Since $d_{1}=d_{1}^{\prime}, d_{2}=d_{2}^{\prime}$ and $d_{3}=d_{3}^{\prime} \geqslant d_{p}^{\prime} \geqslant 4, G$ contains some surprising vertex according to Theorem 18 and Theorem 23.

Finally, we will verify the following majorization theorem to the $c$-cyclic graphs for $c \geqslant 4$.

Proposition 29. Let $\pi=\left(d_{1}, d_{2}, \ldots, d_{n}\right)$ and $\pi^{\prime}=\left(d_{1}^{\prime}, d_{2}^{\prime}, \ldots, d_{n}^{\prime}\right)$ be two different $c$ cyclic degree sequences, and let $G$ and $G^{\prime}$ be the extremal c-cyclic graphs of $\Gamma(\pi)$ and $\Gamma\left(\pi^{\prime}\right)$, respectively. Suppose $\pi \triangleleft \pi^{\prime}, d_{1}=d_{1}^{\prime}$ and $c \geqslant 4$. If there exists some $t$ such that $d_{t}^{\prime} \geqslant c-1$ and $d_{i}=d_{i}^{\prime}$ holds for all $1+t \leqslant i \leqslant n$, then $\rho(G)<\rho\left(G^{\prime}\right)$ and $\mu(G)<\mu\left(G^{\prime}\right)$. 
Proof. Note that $d_{t}^{\prime} \geqslant c-1$ and $d_{i}=d_{i}^{\prime}$ holds for all $1+t \leqslant i \leqslant n$. Then, $d_{q}=d_{q}^{\prime}+1 \geqslant$ $d_{t}^{\prime}+1 \geqslant c \geqslant 4$. If $d_{n}=2$, then $d_{1}^{\prime} \geqslant d_{p}^{\prime}=d_{p}+1 \geqslant d_{q}+1 \geqslant c+1$. Recall that $d_{q}^{\prime} \geqslant c-1 \geqslant 3$. Thus, $2(n+c-1)=\sum_{i=1}^{n} d_{i}^{\prime} \geqslant 2(c+1)+3+2(n-3)=2 n+2 c-1$, a contradiction. Thus, we may suppose that $d_{n}=1$.

If $v_{q} \in V(G) \backslash V\left(\mathcal{R}(G)\right.$ ), then $G$ contains some surprising vertex (since $d_{q} \geqslant 4$ ). Thus, we may suppose that $v_{q} \in V(\mathcal{R}(G))$ in the following. By Proposition 12 and $f\left(v_{p}\right) \geqslant f\left(v_{q}\right)$, it follows that $v_{p} \in V(\mathcal{R}(G))$. If there exists some $x \in N\left(v_{q}\right)$ such that $x \in$ $V(G) \backslash V(\mathcal{R}(G))$, it is easy to see that $x$ is a surprising vertex of $G$. If $N\left(v_{q}\right) \subseteq V(\mathcal{R}(G)$ ), then $d_{\mathcal{R}(G)}\left(v_{1}\right) \geqslant d_{\mathcal{R}(G)}\left(v_{p}\right) \geqslant d_{\mathcal{R}(G)}\left(v_{q}\right)=d_{G}\left(v_{q}\right) \geqslant c$ by Theorem 10 and Proposition 13 . Thus,

$$
2(|V(\mathcal{R}(G))|+c-1)=\sum_{w \in V(\mathcal{R}(G))} d_{\mathcal{R}(G)}(w) \geqslant 3 c+2(|V(\mathcal{R}(G))|-3),
$$

which implies that $c=4$ by $c \geqslant 4$.

By inequality (4) and $d_{1}=d_{1}^{\prime}$, we can conclude that $p=2, q=3, d_{\mathcal{R}(G)}\left(v_{1}\right)=$ $d_{\mathcal{R}(G)}\left(v_{2}\right)=d_{\mathcal{R}(G)}\left(v_{3}\right)=4$ and $d_{\mathcal{R}(G)}(w)=2$ holds for $w \in V(\mathcal{R}(G)) \backslash\left\{v_{1}, v_{2}, v_{3}\right\}$. If there exists some $x \in N_{\mathcal{R}(G)}\left(v_{3}\right) \backslash\left\{v_{1}, v_{2}\right\}$ such that $x \notin N_{\mathcal{R}(G)}\left(v_{2}\right)$, then $x$ is a surprising vertex of $G$. Now, we assume that $N_{\mathcal{R}(G)}\left(v_{3}\right) \backslash\left\{v_{1}, v_{2}\right\}=N_{\mathcal{R}(G)}\left(v_{2}\right) \backslash\left\{v_{1}, v_{3}\right\}$.

Since $c=4$ and $d_{\mathcal{R}(G)}(w)=2$ holds for $w \in V(\mathcal{R}(G)) \backslash\left\{v_{1}, v_{2}, v_{3}\right\}$, by Proposition 15 , we have $v_{2} v_{3} \in E(G)$ and $v_{4} v_{5} \in E(G)$. Choose $y \in N_{\mathcal{R}(G)}\left(v_{3}\right) \backslash\left\{v_{1}, v_{2}\right\}$. By Theorem 10 and Proposition 13, $f\left(v_{3}\right)>f\left(v_{5}\right)$ and $f\left(v_{4}\right) \geqslant f(y)$. Let $G^{*}=G+v_{3} v_{4}+v_{5} y-v_{3} y-v_{4} v_{5}$. By Corollary $6(1), f\left(v_{4}\right)<f(y)$, a contradiction.

\section{Acknowledgements}

The author would like to thank Professor Slobodan K. Simic and the anonymous referee for their valuable comments, corrections and suggestions, which lead to an improvement of the original manuscript.

\section{References}

[1] F. Belardo, E. M. Li Marzi, S. K. Simić, and J. F. Wang. On the spectral radius of unicyclic graphs with prescribed degree sequence. Linear Algebra Appl., 432:23232334, 2010.

[2] T. Bıyıkoğlu and J. Leydold. Graphs with given degree sequence and maximal spectral radius. Electron. J. Combin., 15(1):R119, 2008.

[3] D. M. Cvetković. Some possible directions in further investigations of graph spectra. In Algebraic Methods in Graph Theory, Vol. I, II (Szeged, 1978), pp. 47-67. NorthHolland, Amsterdam-New York, 1981.

[4] R. A. Horn and C. R. Johnson. Matrix Analysis. Cambridge University Press, New York, 1990. 
[5] Y. F. Huang, B. L. Liu, and Y. L. Liu. The signless Laplacian spectral radius of bicyclic graphs with prescribed degree sequences. Discrete Math., 311:504-511, 2011.

[6] X. Y. Jiang, Y. L. Liu, and B. L. Liu. A further result on majorization theorem. Linear Multilinear Algebra, 59:957-967, 2011.

[7] J. S. Li and X. D. Zhang. On the Laplacian eigenvalues of a graph. Linear Algebra Appl., 285:305-307, 1998.

[8] M. H. Liu and B. L. Liu. Some results on the majorization theorem of connected graphs. Acta Math. Sin. (Engl. Ser.), 28:371-378, 2012.

[9] M. H. Liu and B. L. Liu. New method and new results on the order of spectral radius. Comput. Appl. Math., 63:679-686, 2012.

[10] M. H. Liu, B. L. Liu, and Z. F. You. The majorization theorem of connected graphs. Linear Algebra Appl., 431:553-557, 2009.

[11] Y. L. Liu and B. L. Liu. The spectral radius of bicyclic graphs with prescribed degree sequences. Linear Algebra Appl., 433:1015-1023, 2010.

[12] A. W. Marshall and I. Olkin. Inequalities: Theory of Majorization and its Applications. Academic Press, New York, 1979.

[13] Y. L. Pan. Sharp upper bounds for the Laplacian graph eigenvalues. Linear Algebra Appl., 355:287-295, 2002.

[14] A. J. Schwenk. Computing the characteristic polynomial of a graph. In R. Bari and F. Harary, editors, Graphs and Combinatorics-Lecture Notes in Mathematics, vol. 406, pp. 153-172. Springer-Verlag, Berlin-Heidelberg-New York, 1974.

[15] B. F. Wu, E. L. Xiao, and Y. Hong. The spectral radius of trees on $k$ pendant vertices. Linear Algebra Appl., 395:343-349, 2005.

[16] X. D. Zhang. The Laplacian spectral radii of trees with degree sequences. Discrete Math., 308:3143-3150, 2008.

[17] X. D. Zhang. The signless Laplacian spectral radius of graphs with given degree sequences. Discrete Appl. Math., 157:2928-2937, 2009. 